\title{
THE B SUBUNITS OF CHOLERA AND ESCHERICHIA COLI HEAT-LABILE TOXINS ENHANCE THE IMMUNE RESPONSES IN MICE ORALLY IMMUNISED WITH A RECOMBINANT LIVE P-FIMBRIAL VACCINE FOR AVIAN PATHOGENIC E. COLI
}

\author{
In-Gyeong OH, Chetan V. JAWALE and John Hwa LEE* \\ College of Veterinary Medicine and Bio-Safety Research Institute, \\ Chonbuk National University, Jeonju 561-756, South Korea
}

(Received 25 May 2013; accepted 2 July 2013)

This study aimed to investigate the adjuvant effect of recombinant attenuated Salmonella expressing cholera toxin B subunit (CTB) and Escherichia coli heat-labile enterotoxin B subunit (LTB) for the P-fimbriae subunit-based vaccine of avian pathogenic E. coli (APEC) in a murine model. The PapA-specific sIgA and $\mathrm{IgG}$ responses were significantly enhanced after immunisation with the Salmonella-PapA vaccine in the presence of CTB or LTB. The group immunised with the Salmonella-LTB strain promoted Th1-type immunity, whereas that immunised with the Salmonella-CTB strain produced Th2-type immunity. We concluded that both Salmonella-CTB and -LTB strains can enhance the immune response to PapA, and that the LTB strain may be a more effective adjuvant for APEC vaccination, which requires higher Th1-type immunity for protection. Thus, our findings provide evidence that immunisation with an adjuvant, LTB, is one of the strategies of developing effective vaccines against P-fimbriated APEC. sponse

Key words: APEC, Salmonella vaccine delivery, CTB, LTB, immune re-

Avian pathogenic Escherichia coli (APEC) belongs to the extraintestinal pathogenic group of $E$. coli. These bacteria cause airsacculitis, omphalitis, peritonitis, salpingitis, synovitis, and coli septicaemia in poultry (Pourbakhsh et al., 1997; Dho-Moulin and Fairbrother, 1999; Vandemaele et al., 2002; Ewers et al., 2004). Several virulence factors, including P-fimbriae, have been associated with APEC which, however, do not occur universally (Dziva and Stevens, 2008; Ewers et al., 2009; Tóth et al., 2012). Among them, the major subunit of P-fimbriae, PapA, appears to be a suitable candidate as a vaccine target against APEC (Oh et al., 2011).

Oral vaccines can promote mucosal immune responses, as well as provide safe, readily acceptable methods for inducing systemic immune responses (Ogra

*Corresponding author; E-mail: johnhlee@jbnu.ac.kr; Phone: 0082 (63) 270-2553; Fax: 0082 (63) 270-3780 
et al., 2001; Zhao et al., 2006). Mucosal immune responses are important for protecting mucosal surfaces against invasion by microbes, blocking the uptake of ungraded antigens, and preventing the development of potentially injurious immune responses to these antigens (Holmgren and Czerkinsky, 2005). However, mucosal immunisations are frequently ineffective due to weak antigen uptake from mucosal surfaces, and a large dose of antigen is required for induction of immune responses against specific antigens (Ogra et al., 2001). Adjuvants are generally added to the antigens to promote higher immune responses (Holmgren et al., 1992).

A number of products of bacterial origin have been tested as mucosal adjuvants, including bacterial toxins such as cholera toxin (CT) and E. coli heatlabile enterotoxin (LT) (Freytag and Clements, 1999; Fingerut et al., 2006). Both CT and LT are composed of one enzymatically active A unit and five B units that bind to ganglioside GM1 expressed on the eukaryotic cell surface. The nontoxic B subunits of CT (CTB) and LT (LTB) have also been used as adjuvants, as they increase antigen uptake from mucosal surfaces, thus increasing the humoral and cellular immune responses (Holmgren et al., 1993; Yamamoto et al., 1997; McCluskie et al., 2001). LTB and CTB proteins have been successfully expressed in different expression systems such as Mycobacterium bovis and Lactobacillus or Bacillus brevis (Slos et al., 1998; Hayward et al., 1999; Goto et al., 2000). Recently, live attenuated Salmonella bacteria were employed for delivery of antigens for mucosal vaccination (Branger et al., 2007, Oh et al., 2011). Salmonella bacteria as a carrier target the mucosal-associated lymphoid tissues in the gut and thereby improve the possibility of stimulating mucosal immunity, which may be important for providing protection against some infectious agents (Sirard et al., 1999). A balanced-lethal host-vector system based on the essential bacterial aspartate $\beta$-semialdehyde dehydrogenase (asd) gene has been used to specify recombinant antigens from the $\mathrm{Asd}^{+}$plasmids pYA3493 and pYA3560 (Nakayama et al., 1998; Kang et al., 2002).

In this study, we constructed recombinant attenuated $S$. Typhimurium strains that express PapA, CTB and LTB. We used mice to evaluate whether oral vaccination with a live attenuated $S$. Typhimurium strain expressing PapA induced stronger immune responses when inoculated with recombinant CTB and LTB or when inoculated with another $S$. Typhimurium strain expressing CTB or LTB as an adjuvant. We also examined the effect of synergy between these adjuvant strains.

\section{Materials and methods}

\section{Bacterial strains, plasmids and growth conditions}

The his $G$ Acrp-28 $\Delta a s d A 16$ attenuated $S$. Typhimurium $\chi 8501$ (Gulig and Curtiss, 1987) was used for the delivery of antigen proteins. Plasmids pYA3493 
and pYA3560 were described previously (Oh et al., 2011). All strains were grown at $37{ }^{\circ} \mathrm{C}$ in Luria-Bertani (LB) media (Difco, Sparks, MD, USA) with or without antibiotics. Diaminopimelic acid (DAP) (Sigma, St Louis, MO, USA) was added for the growth of $\mathrm{Asd}^{-}$strains (Nakayama et al., 1998). Phosphatebuffered saline containing $0.01 \%$ gelatine was used for the suspension of the vaccine strains.

\section{Construction of PapA vaccine and adjuvants}

For construction of PapA, LTB, and CTB strains, each gene was obtained by polymerase chain reaction using a pair of primers:

PapA (F, 5'CCGCGAATTCGCTCCAACTATTCCACAG3'; R, 5'CCCGCG TCGACTTACTGGTAACTTAAAT') (GenBank accession no. AF237478.1), LTB (F, 5'CCGCGAATTCGCTCCCCAGTCTATTACAG3'; R, 5'CCGCAAGCT TCTAGTTTTCCATACTGATTG3') (GenBank accession no. AB011677.1), and CTB (F, 5'GAATTCGCTCCCATGGCTTTCGCTAC3'; R, 5'AATCTTACATGT TTGGGCAA3') (GenBank accession no. JQ683136.1). These primers were designed using the DNASTAR Lasergene 5.07/5.52 programme (DNASTAR Inc., USA). The size of PCR products for the PapA, LTB and CTB genes were 486, 327 and 408 base pairs, respectively. The primers used for PCR amplification of the PapA gene carried the restriction digestion sites for EcoRI-SalI, and primers for $C T B$ and $L T B$ carried EcoRI and HindIII sites. The asd ${ }^{+}$pYA3493 and pYA3560 were used as backbone plasmids. The amplified PCR products of PapA and CTB were cloned in plasmid pYA3493, and the LTB product was cloned in pYA3560. The presence of inserts in these plasmids was confirmed by restriction digestion with respective enzymes. These cloned plasmids were individually introduced into $\operatorname{asd}^{-} \chi 6212$, to obtain the balanced-lethal E. coli constructs. The purified plasmids obtained from the balanced-lethal E. coli constructs were then electroporated into the attenuated $S$. Typhimurium strain $\chi 8501$, and resulted in strains JOL713 (PapA), JOL757 (CTB) and JOL 758 (LTB), respectively. Selection for transformants was achieved by growth on LB agar plates without DAP supplementation. Only clones containing the recombinant plasmids were able to grow under these conditions.

\section{Western blot analyses}

Secreted antigen proteins from recombinant strains were obtained from cell-free culture supernatants by the TCA-precipitation process (Oh et al., 2011). Attenuated Salmonella containing the pYA3493 vector was used as control. Protein samples were separated by sodium dodecyl sulphate-polyacrylamide gel electrophoresis (SDS-PAGE) and transferred to $0.2 \mu \mathrm{m}$ microporous polyvinylidene fluoride membranes (Millipore, Bedford, MA, USA) for Western blot analysis. Membranes were probed with polyclonal rabbit antisera (1:500 dilu- 
tion) specific for each antigen. The secondary antibody, the goat anti-rabbit HRP conjugated antibody (Southern Biotech, USA) was used for detection of recombinant protein at dilution of 1:5000.

\section{Immunisation and collection of samples}

Groups of 6-week-old female BALB/c mice were immunised with Salmonella-PapA vaccine, JOL 713 alone or in combination with the LTB, JOL 758 and/or CTB, JOL 757 strains (Table 1). There were 10 mice in each group. All the mice were maintained at an antibiotic-free autoclaved feed and water. A total of $20 \mu \mathrm{l}$ containing $2 \times 10^{9}$ colony-forming units of bacterial cells were orally administered. Sera were obtained by retro-orbital puncture in heparinised capillary tubes and centrifuged at $4,000 \times g$ for $5 \mathrm{~min}$. Vaginal samples were collected by washing the vaginal cavity with $100 \mu \mathrm{l}$ of PBS. All samples were collected over a 14 -week period at 2-week intervals and were stored at $-20{ }^{\circ} \mathrm{C}$ until assessed by ELISA.

Table 1

Experimental groups of mice in this study

\begin{tabular}{ll}
\hline Group & \multicolumn{1}{c}{ Bacterial strain used } \\
\hline A & JOL713 (PapA ) \\
B & JOL713 + JOL757 (CTB) \\
C & JOL713 + JOL758 (LTB) \\
D & JOL713 + JOL757 + JOL758 \\
\hline
\end{tabular}

\section{Determination of antibody titres}

PapA-specific antibodies in the serum and vaginal samples were detected by enzyme-linked immunosorbent assay (ELISA). Polystyrene 96-well flatbottom microtitre plates were coated with $1 \mu \mathrm{g}$ of purified PapA per well and blocked with PBS containing $3 \%$ skim milk at $4{ }^{\circ} \mathrm{C}$ overnight. Vaginal secretions and sera were diluted 1:4 and 1:100, respectively. A 100- $\mu 1$ volume of diluted sample was added to individual wells in duplicate and incubated for 1.5-2 $\mathrm{h}$ at $37^{\circ} \mathrm{C}$. Plates were treated with horseradish peroxidase-conjugated goat antimouse IgG, IgG1 or IgG2a (Southern Biotechnology Inc., Birmingham, AL, USA) for sera and IgA for vaginal secretions. The reactions were visualised with $o$-phenylenediamine (Sigma) and stopped with $\mathrm{H}_{2} \mathrm{SO}_{4}$. The absorbance in each well was measured using an automated ELISA spectrophotometer (TECAN, Salzburg, Austria) at a wavelength of $492 \mathrm{~nm}$. The concentrations of antibodies were determined by comparing results of two concentrations of the standard mouse IgG (Southern Biotech). 


\section{Statistical analysis}

Independent-samples $t$-test was used to determine significant differences in antibody titres between immunised and control groups and significant differences in antibody titres of IgG2a and IgG1 in immunised groups. Statistical results were considered significant when $P$ values were $<0.05$. All statistical analyses were carried out with the SPSS 16.0 programme (SPSS Inc.).

\section{Results}

\section{Expression of PapA, CTB and LTB in attenuated S. Typhimurium}

The papA, $C T B$ and $L T B$ genes were cloned into expression vector as described in the Materials and methods, and the expression of each protein was determined by conducting Western blot analysis with specific antibodies against each of the proteins. The strains produced the PapA, LTB and CTB at approximate molecular weights of $20.3 \mathrm{kDa}, 11 \mathrm{kDa}$ and $13.6 \mathrm{kDa}$, respectively, and large amounts of these proteins were detected in the culture supernatant (Fig. 1). Little or no protein was detected in the control supernatant.

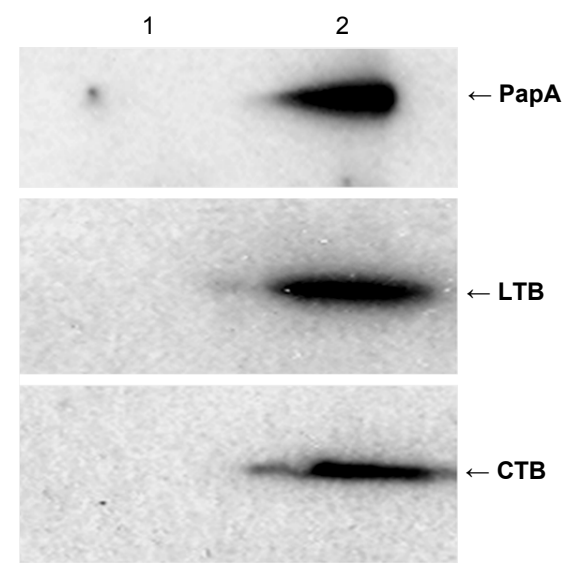

Fig. 1. Protein expression as measured by Western blot analysis. Each protein is indicated by an arrow. Molecular sizes in kilodaltons are PapA, 20.3; LTB, 11; CTB, 13.6. Lanes: 1, control containing pYA3493 only; 2, PapA, LTB and CTB strains

\section{Serum anti-PapA IgG responses}

The induction of plasma IgG responses against the purified PapA antigen was monitored in the weeks after vaccination. At week 1, groups immunised in conjunction with CTB and LTB produced similar PapA-specific IgG antibodies as the non-adjuvant group. At week 2, groups immunised in conjunction with 
CTB and LTB (Group B and Group C, respectively) produced about 2.4 times higher levels of PapA-specific IgG antibodies compared to the non-adjuvant group (Group A) (Fig. 2A). However, the group immunised in conjunction with both adjuvants (Group D) produced similar PapA-specific IgG antibodies as the non-adjuvant group. After week 10, the antibody titres in Group B and Group C continued to increase, but a slight decrease was observed in Group A and Group D, immunized with both CTB and LTB. Over the course of the experimental period, antibody titres were observed in the order Group C > Group B $>$ Group A > Group D.
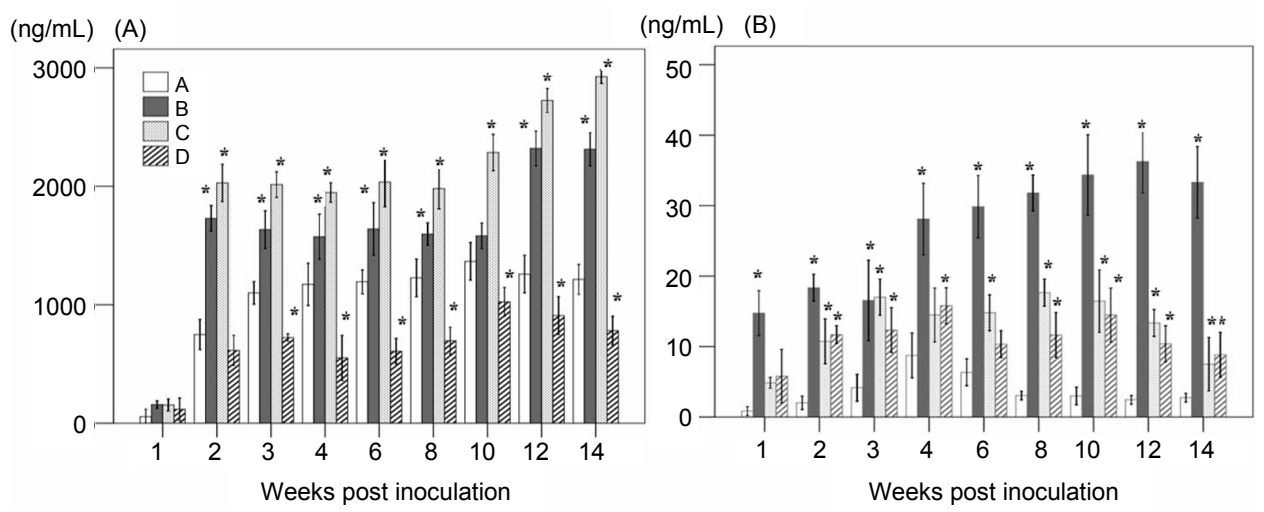

Fig. 2. Anti-PapA antibody titres in serum (A) and vaginal samples (B) during the observed periods. Mice were immunised as shown in Table 1. Group A, immunisation with PapA alone; Group B, immunisation with PapA combined with CTB; Group C, immunisation with PapA

combined with LTB; Group D, immunisation with PapA combined with CTB and LTB.

Data are the mean of all mice in each group; error bars show SD. Asterisks indicate a significant difference between the values of Groups B, C and D mice $\left({ }^{*} \mathrm{P}<0.05\right)$ and those of Group A mice

\section{Mucosal anti-PapA sIgA responses}

In order to evaluate the mucosal immune responses induced after vaccination, the PapA protein specific sIgA titres were estimated from the vaginal wash samples. The sIgA titres in all adjuvant groups (Groups B, C and D) were higher than those in the non-adjuvant group, Group A (Fig. 2B). Overall, Group B with CTB generated the highest titre among the groups. At week 4, the antibody titre in each of Groups A, C and D reached its peak. Thereafter, titres in Group A declined rapidly, but the decrease was slow in Groups $\mathrm{C}$ and D. The IgA titres in Group B continued to increase and were around 4 times higher than in other groups. These data indicate that the inclusion of CTB adjuvant with the vaccine candidate enhances the production of mucosal immune responses. 


\section{PapA-specific IgG1 and IgG2a subtypes}

To further evaluate the nature of immunity following immunisation, we determined the levels of IgG isotype subclasses IgG2a and IgG1 at week 12 after immunisation. Different trends were observed depending on the types of adjuvants used (Fig. 3). Group B with CTB produced about 4 times higher level of IgG1 isotype compared to the other groups; however, Group C with LTB produced more IgG2a isotype than either Group B or Group D, and in Group D a higher level of IgG2a isotype production was induced than in Group A. The IgG2a/IgG1 ratios in Groups A, B, C and D were approximately 2.0, 0.5, 9.1 and 5.8 , respectively $(\mathrm{P}<0.05)$.

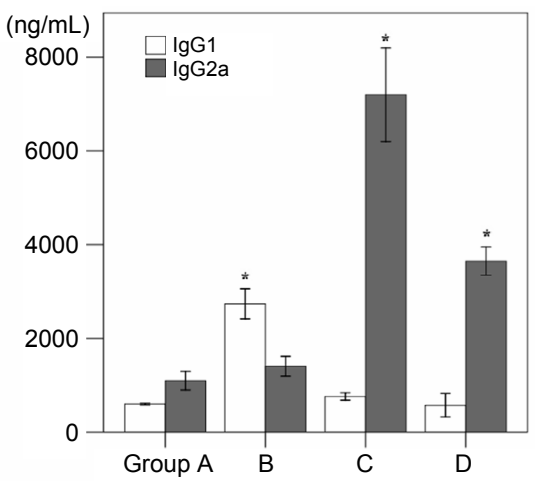

Fig. 3. PapA-specific IgG1 and IgG2a responses. Immunisation groups were Group A, immunisation with PapA; Group B, immunisation with PapA combined with CTB; Group C, immunisation with PapA combined with LTB; Group D, immunisation with PapA combined with CTB and LTB Data are the mean of all mice in each group; error bars show SD. Asterisks indicate a significant difference between the values of Groups B, C and D mice $\left({ }^{*} \mathrm{P}<0.05\right)$ and those of Group A mice

\section{Discussion}

The induction of immune responses following mucosal immunisation usually depends on the co-administration of appropriate adjuvants (Zhang et al., 2008). Adjuvants are substances that, when co-administered with antigen, can modulate antigen-specific immune responses. A few adjuvants have been examined for viral infections such as Newcastle disease in chickens (Zhang et al., 2007). However, few studies of adjuvants have yet been performed for bacterial infections of chickens.

The mucosal adjuvants most commonly used in animal models are cholera toxin (CT), E. coli heat-labile enterotoxin (LT) and their derivatives (Freytag and Clements, 2005). Mucosal adjuvants such as LT and CT are highly effective but are generally considered to be potent enterotoxins. Efforts to develop less toxic versions that would be safe for use have focused on the use of the non-toxic B 
subunit, which lacks enzymatic activity (Yamamoto et al., 1997; McCluskie et al., 2001). In some studies, mucosal co-immunisation with CTB or LTB has been shown to enhance the systemic and mucosal immune responses to co-administered antigens (Isaka et al., 1999; de Haan et al., 2001). Similarly, our study showed that PapA-specific serum IgG and mucosal IgA titres increased significantly when mice were administered Salmonella-PapA vaccine in the presence of SalmonellaLTB or -CTB. Rapid declines in immune responses during the entire experimental period were observed in mice immunised without adjuvant. Particularly, groups with LTB had much higher induction of serum IgG titres compared to other groups in this study. Previous reports have suggested that LTB is markedly enhanced in the mucosal and systemic immune response to various foreign antigens (Verweij et al., 1998; Fingerut et al., 2006). LTB, which has non-toxic activity, is responsible for binding to the monosialotetrahexosylganglioside (GM1) ganglioside-receptor of target cells to take up whole toxin units within the cell. LTB acts to modulate cell-mediated responses (Th1) to humoral responses (Th2) (Simmons et al., 2001). LTB enhances the uptake of co-administered proteins (Freytag and Clements, 2005) and both mucosal and cellular immune responses (Fingerut et al., 2006). In addition, LTB is highly pH- and protease-resistant; it resists degradation during transit through the gastrointestinal system (Ruddock et al., 1995), making LTB an effective adjuvant for mucosal immunisation.

Th1-type responses in mice are generally associated with enhanced levels of IgG2a antibodies, which are thought to have superior neutralising capabilities, and cytotoxic T lymphocyte (CTL), which are desirable for protection against intracellular bacterial pathogens (Strindelius et al., 2004). Th2-type immune responses provide B-cell antibody production and promote class switching to IgG1 (McCluskie et al., 2001; Strindelius et al., 2004). Our results showed that the Salmonella-CTB strain produced more IgG1 isotypes indicating Th2-type responses, while the Salmonella-LTB strain produced more IgG2a isotypes indicating Th1-type responses. These results are similar to those of other reports suggesting that the Salmonella delivery system induces a predominance of Th1 type responses against both passenger and Salmonella antigens (Vindurampulle et al., 2004). Th1-dominated cell-mediated responses are likely to be more important for clearing P-fimbriated APEC, believed to survive and multiply within macrophages (Wigley et al., 2005; Chappell et al., 2009). Thus, these results indicated that the Salmonella-LTB strain may be a more effective adjuvant against APEC, which survives inside macrophages (Vindurampulle et al., 2004; Bastiani et al., 2005).

A previous study addressed potential synergy between different adjuvants (McCluskie and Davis, 1998). Our present data, however, showed that no synergistic effect was observed after administration of the combination of LTB and CTB. PapA-specific sIgA responses in the group immunised with both CTB and LTB were lower than those in the individual CTB or LTB groups. The principal receptor for both CTB and LTB is GM1-ganglioside, a glycosphingolipid found 
ubiquitously on the surface of mammalian cells (Freytag and Clements, 2005). Thus, further studies could elucidate whether CTB and LTB would compete for this receptor, causing decreased immune response. In the light of multiple alternative virulence mechanisms mediating the pathogenicity of APEC, investigations are also needed for inclusion of further virulence antigens into the recombinant live vaccine candidate described in this study.

\section{Acknowledgements}

This work was supported by the Mid-career Researcher Program through the National Research Foundation (NRF) grant funded by the Ministry of Education, Science and Technology (MEST) (No. 2012-R1A2A4A01002318), Republic of Korea, and was also supported by research funds of Chonbuk National University in 2013.

\section{References}

Bastiani, M., Vidotto, M. C. and Horn, F. (2005): An avian pathogenic Escherichia coli isolate induces caspase 3/7 activation in J774 macrophages. FEMS. Microbiol. Lett. 253, 133-140.

Branger, C. G., Fetherston, J. D., Perry, R. D. and Curtiss, R. 3rd (2007): Oral vaccination with different antigens from Yersinia pestis KIM delivered by live attenuated Salmonella typhimurium elicits a protective immune response against plague. Adv. Exp. Med. Biol. 603, 387-399.

Chappell, L., Kaiser, P., Barrow, P., Jones, M. A., Johnston, C. and Wigley, P. (2009): The immunobiology of avian systemic salmonellosis. Vet. Immunol. Immunopathol. 128, 53-59.

Dho-Moulin, M. and Fairbrother, J. M. (1999): Avian pathogenic Escherichia coli (APEC). Vet. Res. 30, 299-316.

de Haan, L., Verweij, W. R., Feil, I. K., Holtrop, M., Brands, R., van Scharrenburg, G. J. M., Palache, A. M., Agsteribbe, E. and Wilschut, J. (2001): Nasal or intramuscular immunization of mice with influenza subunit antigen and the B subunit of Escherichia coli heat-labile toxin induces IgA-or IgG-mediated protective mucosal immunity. Vaccine 19, 2898-2907.

Dziva, F. and Stevens, M. P. (2008): Colibacillosis in poultry: unraveling the molecular basis of virulence of avian pathogenic Escherichia coli in their natural hosts. Avian Pathol. 37, $355-366$.

Ewers, C., Antão, E. M., Diehl, I., Philipp, H. C. and Wieler, L. H. (2009): Intestine and environment of the chicken as reservoirs for extraintestinal pathogenic Escherichia coli strains with zoonotic potential. Appl. Environ. Microbiol. 75, 184-192.

Ewers, C., Janben, T., Kiebling, S., Philipp, H. C. and Wieler, L. H. (2004): Molecular epidemiology of avian pathogenic Escherichia coli (APEC) isolated from colisepticemia in poultry. Vet. Microbiol. 104, 91-101.

Fingerut, E., Gutter, B., Goldway, M., Eliahoo, D. and Pitcovski, J. (2006): B subunit of E.coli enterotoxin as adjuvant and carrier in oral and skin vaccination. Vet. Immunol. Immunopathol. 112, 253-263.

Freytag, L. C. and Clements, J. D. (1999): Bacterial toxins as mucosal adjuvants. Curr. Top. Microbiol. Immunol. 236, 215-236.

Freytag, L. C. and Clements, J. D. (2005): Mucosal adjuvants. Vaccine 23, 1804-1813. 
Goto, N., Maeyama, J. I., Yasuda, Y., Isaka, M., Matano, K., Kozuka, S., Taniguchi, T., Miura, Y., Okhuma, K. and Tochikubo, K. (2000): Safety evaluation of recombinant cholera toxin B subunit produced by Bacillus brevis as a mucosal adjuvant. Vaccine 18, 2164-2171.

Gulig, P. A. and Curtiss, R. 3rd (1987): Plasmid-associated virulence of Salmonella Typhimurium. Infect. Immun. 55, 2891-2901.

Hayward, C. M. M., O'Gaora, P., Young, D. B., Griffin, G. E., Thole, J., Hirst, T. R., CastelloBranco, L. R. R. and Lewis, D. J. M. (1999): Construction and murine immunogenicity of recombinant Bacille Calmette Guerin vaccine expressing the B subunit of Escherichia coli heat labile enterotoxin. Vaccine 17, 1272-1281.

Holmgren, J. and Czerkinsky, C. (2005): Mucosal immunity and vaccines. Nat. Med. 11, S45-S53.

Holmgren, J., Czerkinsky, C., Lycke, N. and Svennerholm, A-M. (1992): Mucosal immunity: implication for vaccine development. Immunobiology 184, 157-179.

Holmgren, J., Lycke, N. and Czerkinsky, C. (1993): Cholera toxin and cholera B subunit as oralmucosal adjuvant and antigen vector systems. Vaccine 11, 1179-1184.

Isaka, M., Yasuda, Y., Kozuka, S., Tanguchi, T., Matano, K., Maeyama, J., Komiya, T., Ohkuma, K., Goto, N. and Tochikubo, K. (1999): Induction of systemic and mucosal antibody responses in mice immunized intranasally with aluminium-non-adsorbed diphtheria toxoid together with recombinant cholera toxin B subunit as an adjuvant. Vaccine 18, 743-751.

Kang, H. Y., Srinivasan, J. and Curtiss, R. 3rd (2002): Immune responses to recombinant pneumococcal PspA antigen delivered by live attenuated Salmonella enterica serovar Typhimurium vaccine. Infect. Immun. 70, 1739-1749.

McCluskie, M. J. and Davis, H. L. (1998): CpG DNA is a potent enhancer of systemic and mucosal immune responses against hepatitis B surface antigen with intranasal administration to mice. J. Immunol. 161, 4463-4466.

McCluskie, M. J., Weeratna, R. D., Clements, J. D. and Davis, H. L. (2001): Mucosal immunization of mice using CpG DNA and/or mutants of the heat-labile enterotoxin of Escherichia coli as adjuvants. Vaccine 19, 3759-3768.

Nakayama, K., Kelly, S. M. and Curtiss, R. 3rd (1998): Construction of an Asd ${ }^{+}$expression-cloning vector: stable maintenance and high level expression of cloned genes in a Salmonella vaccine strain. Nature Biotechnol. 6, 693-697.

Ogra, P. L., Faden, H. and Welliver, R. C. (2001): Vaccination strategies for mucosal immune responses. Clin. Microbiol. Rev. 14, 430-445.

Oh, I. G., Moon, B. M. and Lee, J. H. (2011): Induction of systemic and mucosal immune responses in mice orally administered with recombinant attenuated Salmonella expressing subunits of P fimbriae of avian pathogenic Escherichia coli. J. Vet. Clin. 28, 297-302.

Pourbakhsh, S. A., Boulianne, M., Martineau-Doize, B. and Fairbrother, J. M. (1997): Virulence mechanisms of avian fimbriated Escherichia coli in experimentally inoculated chickens. Vet. Microbiol. 58, 195-213.

Ruddock, L. W., Ruston, S. P., Kelly, S. M., Price, N. C., Freedman, R. B. and Hirst, T. R. (1995): Kinetics of acid-mediated disassembly of the B subunit pentamer of Escherichia coli heatlabile enterotoxin. Molecular basis of pH stability. J. Biol. Chem. 270, 29953-29958.

Simmons, C. P., Ghaem-Magami, M., Petrovska, L., Lopes, L., Chain, B. M., Williams, N. A. and Dougan, G. (2001): Immunomodulation using bacterial enterotoxins. Scand. J. Immunol. 53, 218-226.

Sirard, J. C., Niedergang, F. and Kraehenbuhl, J. P. (1999): Live attenuated salmonella: a paradigm of mucosal vaccine, Immunol. Rev. 171, 5-26.

Slos, P., Dutot, P., Reymund, J., Kleinpeter, P., Prozzi, D., Kieny, M. P., Delcour, J., Mercenier, A. and Hols, P. (1998): Production of cholera toxin B subunit in Lactobacillus. FEMS Microbiol. Lett. 169, 29-36.

Strindelius, L., Filler, M. and Sjöholm, I. (2004): Mucosal immunization with purified flagellin from Salmonella induces systemic and mucosal immune responses in $\mathrm{C} 3 \mathrm{H} / \mathrm{HeJ}$ mice. Vaccine 22, 3797-3808. 
Tóth, I., Dobrindt, U., Koscsó, B., Kósa, A., Herpay, M. and Nagy, B. (2012): Genetic and phylogenetic analysis of avian extraintestinal and intestinal Escherichia coli. Acta Microbiol. Immunol. Hung. 59, 393-409.

Vandemaele, F., Assadzadeh, A., Derijcke, J., Vereecken, M. and Goddeeris, B. M. (2002): Avian pathogenic Escherichia coli (APEC). Tijdschr. Diergeneeskd. 127, 582-588.

Verweij, W. R., de Haan, L., Holtrop, M., Agsteribbe, E., Brands, R., van Scharrenburg, G. J. M. and Wilschut, J. (1998): Mucosal immunoadjuvant activity of recombinant Escherichia coli heat-labile enterotoxin and its B subunit: Induction of systemic IgG and secretory IgA responses in mice by intranasal immunization with influenza virus surface antigen. Vaccine 16, 2069-2076.

Vindurampulle, C. J., Cuberos, L. F., Barry, E. M., Pasetti, M. F. and Levine, M. M. (2004): Recombinant Salmonella enterica serovar Typhi in a prime-boost strategy. Vaccine 22, 3744-3750.

Wigley, P., Hulme, S., Powers, C., Beal, R., Smith, A. and Barrow, P. (2005): Oral infection with the Salmonella enterica serovar Gallinarum 9R attenuated live vaccine as a model to characterise immunity to fowl typhoid in the chicken. BMC Vet. Res. 1, 2. doi:10.1186/17466148-1-2

Yamamoto, S., Kiyono, H., Yamamoto, M., Imaoka, K., Fujihashi, K., Van Ginkel, F. W., Noda, M., Takeda, Y. and McGhee, J. R. (1997): A nontoxic mutant of cholera toxin elicits Th2type responses for enhanced mucosal immunity. Proc. Natl Acad. Sci. USA 13, 5267-5272.

Zhang, L., Zhang, M., Li, J., Cao, T., Tian, X. and Zhou, F. (2008): Enhancement of mucosal immune responses by intranasal co-delivery of Newcastle disease vaccine plus $\mathrm{CpG}$ oligonucleotide in SPF chickens in vivo. Res. Vet. Sci. 85, 495-502.

Zhang, X., Zhang, X. and Yang, Q. (2007): Effect of compound mucosal immune adjuvant on mucosal and systemic immune responses in chicken orally vaccinated with attenuated Newcastle-disease vaccine. Vaccine 30, 3254-3262.

Zhao, X., Zhang, M., Li, Z. and Frankel, F. R. (2006): Vaginal protection and immunity after oral immunization of mice with a novel vaccine strain of Listeria monocytogenes expressing human immunodeficiency virus type 1 gag. J. Virol. 80, 8880-8890. 